\title{
Optical Absorption and ESR Studies of Poly(p-phenylene sulfide) Doped with Sulfur Trioxide
}

\author{
Hiroshi ShImIZU, Hisaaki Kanetsuna, and Yoshikazu TANABE \\ Research Institute for Polymers and Textiles, \\ 1-1-4 Higashi, Yatabe-machi, Tsukuba-gun, \\ Ibaraki 305, Japan
}

(Received December 8, 1986)

\begin{abstract}
Optical absorption and ESR spectra of $\mathrm{SO}_{3}$-doped poly(p-phenylene sulfide) (PPS) with relatively high conductivity were measured. Two absorption bands were observed at 0.9 and $3.1 \mathrm{eV}$ at the initial doping state. The intensity of these two bands increased with the rise of doping level. ESR spectra with a $g$-value of 2.0075 were observed for the PPS-SO $\mathrm{S}_{3}$ system at the same doping level as that of absorption spectra. The intensity of ESR signals increased with the doping level, while the linewidth showed a nearly constant value of 3.6 gauss. The spin density of the PPS- $-\mathrm{SO}_{3}$ system increased monotonously with the $\mathrm{SO}_{3}$ concentration. In both cases of optical absorption and ESR spectra of $\mathrm{SO}_{3}$-doped PPS, the spectral intensity increased slightly under UVlight irradiation, while these spectra were little affected by the near IR-light irradiation. These spectroscopic results suggest the presence of sulfur-centered radical-cations rather than a bipolaron state. Two intense bands of the PPS- $\mathrm{SO}_{3}$ system observed at 0.9 and $3.1 \mathrm{eV}$ were considered to be assigned to electronic transitions from low lying occupied bands of PPS to the singly occupied highest band.
\end{abstract}

KEY WORDS Optical Absorption / ESR / Poly( $p$-phenylene sulfide)

Conductive Polymer / Doping Mechanism / Electronic Structure /

Conduction Mechanism /

Various conductive polymers such as polyacetylene (PA), poly( $p$-phenylene) (PPP), polypyrrole (PPy), and polythiophene (PT) have received considerable attention. Many investigations on the conduction mechanisms and practical applications of these materials have been actively performed to date. Poly ( $p$ phenylene sulfide) (PPS) has the advantage of being melt and solution processible. The conduction mechanism is of much interest because of its non-conjugated structure. Frommer et $a l .{ }^{1}$ have recently reported a high conductivity $\left(200 \mathrm{~S} \mathrm{~cm}^{-1}\right)$ for $\mathrm{AsF}_{5}$-doped PPS film cast from $\mathrm{AsF}_{3}$ solution. For the conduction mechanism of heavily $\mathrm{AsF}_{5}$-doped PPS, several ideas such as the interchain crosslinking ${ }^{2}$ and the intramolecular linkage resulting in the formation of benzothiophene structure ${ }^{3}$ have been proposed from the results of elementary analyses and IR spectra. An optical absorption study ${ }^{4}$ of $\mathrm{AsF}_{5}$-doped PPS has shown the transitions which are assigned to polaron and bipolaron states, formed within the $\pi \rightarrow \pi^{*}$ band gap. The electronic structure of doped PPS is unstable in the atmosphere ${ }^{5}$ and an XPS study of doped PPS after exposing to air showed the presence of various doping mechanisms depending on dopants. ${ }^{6}$

In this paper we mention the in situ measurements of optical absorption and ESR spectra of PPS doped with sulfur trioxide $\left(\mathrm{SO}_{3}\right)$. Optical absorption spectra are not inconsistent with the bipolaron model but ESR spectra are inconsistent with it. To explain this discrepancy, the change of these spectra under photoirradiation was observed. The electronic struc- 
ture of the PPS- $\mathrm{SO}_{3}$ system is discussed based on these spectroscopic results.

\section{EXPERIMENTAL}

Thin transparent films of PPS $(15-25 \mu \mathrm{m}$ thick) from Kureha Chemical Industry Co., Ltd. were doped with $\mathrm{SO}_{3}$ in the vapor phase under a pressure of $c a$. 4 Torr at room temperature. The DC conductivities for $\mathrm{SO}_{3}$ doped PPS were measured in situ at room temperature by the standard four probe technique. The dopant content was evaluated from the increase in the weight of PPS films after doping. The optical absorption and ESR spectra of $\mathrm{SO}_{3}$-doped PPS were measured in a sealed quartz cell at room temperature using a Cary 17D spectrophotometer and a JES-FE3X ESR spectrophotometer, respectively, after evacuating an excess amount of dopant. The $g$ value and the peak-to-peak linewidth $\left(\Delta H_{\mathrm{pp}}\right)$ of ESR signals were determined by using a standard sample of $\mathrm{Mn}^{2+} / \mathrm{MgO}$. The modulation width was kept within $25 \%$ of $\Delta H_{\mathrm{pp}}$. The spin density of $\mathrm{SO}_{3}$-doped PPS was determined by a quantitative comparison of double integration of the ESR signal with that of 4-oxo-2,2,6,6-tetramethylpyperidine-1oxyl. A $1 \mathrm{~kW}$ xenon lamp (WACOM Co., Ltd.) was used as the light source for measurements of absorption and ESR spectra under photoirradiation. To control the increase in temperature of the sample during photoirradiation, the quartz cells filled with distilled water were put between the light source and sample. UV (300-400 nm) and near-IR lights (above $800 \mathrm{~nm}$ ) were obtained using a Toshiba UV-D36C filter and a IR-D80A filter, respectively.

\section{RESULTS AND DISCUSSION}

By doping $\mathrm{SO}_{3}$, the $\mathrm{DC}$ conductivities of PPS increased to $c a \cdot 10^{-7} \mathrm{~S} \mathrm{~cm}^{-1}\left(30 \mathrm{~mol}^{\%} \%\right.$ $\left.\mathrm{SO}_{3}\right)$ and $c a \cdot 10^{-5} \mathrm{~S} \mathrm{~cm}^{-1}\left(120 \mathrm{~mol}_{0}^{\circ} \mathrm{SO}_{3}\right)$.

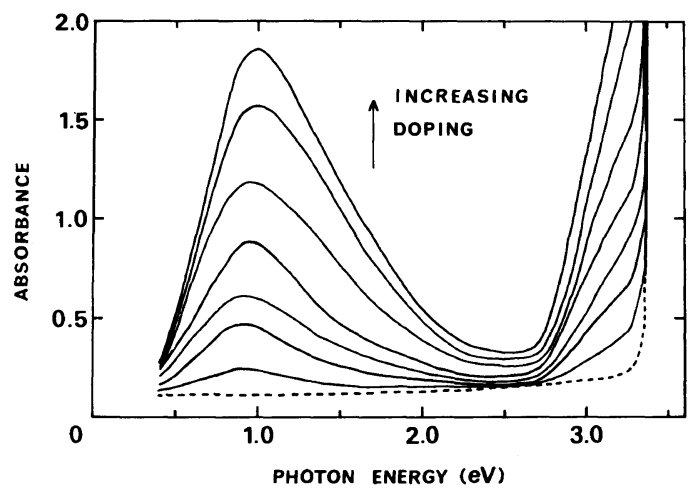

Figure 1. Evolution of the absorption spectrum of PPS as a function of $\mathrm{SO}_{3}$ doping level: the broken line denotes the absorption spectrum of undoped PPS and solid lines show those of $\mathrm{SO}_{3}$-doped PPS. The top curve corresponds to $11.5 \mathrm{~mol}_{\%} \% \mathrm{SO}_{3}$.

Optical Absorption and ESR Spectra of $\mathrm{SO}_{3}$ Doped PPS

Figure 1 shows the evolution of the in situ absorption spectrum of PPS as a function of $\mathrm{SO}_{3}$ doping level. The absorption spectrum of undoped PPS (broken line) exhibits a bandedge at $3.3 \mathrm{eV}$. In the initial stage of doping, the transparent PPS film became greenish yellow and two transitions at 0.9 and $c a .3 .1 \mathrm{eV}$ were observed (solid line). The color of the film changed to blue and the intensity of these transitions increased with the doping level, whereas the peak position of the intense band in the near-IR region shifted from 0.9 to $1.0 \mathrm{eV}$. In this figure, the highest doping level corresponds to the concentration of $11.5 \mathrm{~mol} \%$ $\mathrm{SO}_{3}$. As shown in Figure 1, the oscillator strength of the near-IR band was larger than that of the UV band $(3.1 \mathrm{eV})$. Similar absorption spectra were also observed for $\mathrm{AsF}_{5}$ doped PPS. ${ }^{4,7}$

At the same doping level as that of absorption spectra, in situ ESR spectra of $\mathrm{SO}_{3}$ doped PPS showed a single narrow line with no hyperfine splittings (Fig. 2). The lineshape of ESR signals of $\mathrm{SO}_{3}$-doped PPS was the Lorentzian type. ESR spectra in our doping levels of $\mathrm{SO}_{3}$ showed a $g$-value of 2.0075 . The intensity of ESR signals was enhanced with 


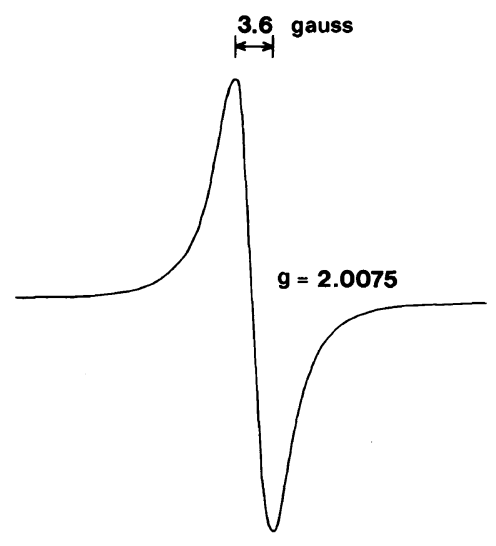

Figure 2. ESR spectrum of $\mathrm{SO}_{3}$-doped PPS $\left(5.13 \mathrm{~mol} \% \mathrm{SO}_{3}\right)$.

increase in the doping level as well as optical absorption, while the linewidth $\left(\Delta H_{\mathrm{pp}}\right)$ showed a nearly constant value of 3.6 Gauss. Shine et $a l .{ }^{8}$ reported the ESR spectrum with a $g$ value of 2.00737 of di-p-tolyl sulfide in $96 \%$ sulfuric acid (green solution), and concluded that the ESR signal of diphenyl sulfide was attributed to the divalent sulfur radical cation, $\stackrel{-{ }^{-}}{-}-$. Therefore, the observed ESR signal with a $g$-value of 2.0075 for $\mathrm{PPS}-\mathrm{SO}_{3}$ system indicate the presence of sulfur-centered radical cation. ${ }^{9,10}$ Two transitions observed at 0.9 and $3.1 \mathrm{eV}$ would be related to the formation of radical-cations on the sulfur atoms of PPS.

Figure 3 shows the dependence of the spin density of the PPS- $\mathrm{SO}_{3}$ system upon the dopant concentration $\left(\mathrm{mol}^{\circ} / \mathrm{SO}_{3}\right)$. As shown in this figure, the spin density increased monotonously with the rise of dopant concentration. This behavior of the spin density for PPS $-\mathrm{SO}_{3}$ system was similar to that previously reported by Kuroiwa et al. ${ }^{11}$ The spin density approached a nearly constant value at a higher doping level and reached $3.47 \times 10^{21}$ spins $^{-1}$ at $105.9 \mathrm{~mol}^{\circ} / \mathrm{SO}_{3}$. Figure 3 also shows the number of spins per PPS monomer unit as a function of dopant concentraion. The number of spins per monomer unit is 0.027 at $10.2 \mathrm{~mol}^{\circ} \%, 0.247$ at $35.3 \mathrm{~mol}^{\circ} \%$, and 0.623 at

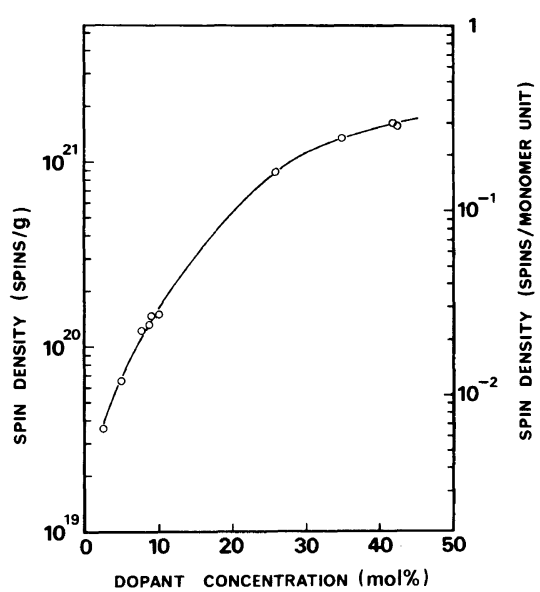

Figure 3. Dependence of the spin density of the PPS$\mathrm{SO}_{3}$ system upon the dopant concentration $\left(\mathrm{mol}_{0} / \mathrm{SO}_{3}\right)$.

$105.9 \mathrm{~mol} \%$, respectively. These results revealed that spins corresponding to $20-25 \%$ of the number of dopants were formed on the PPS at the lower doping level $(\sim 10 \mathrm{~mol} \%)$, and about two spins per three dopant molecules were produced at a higher doping level (above $30 \mathrm{~mol}^{\circ} \%$ ) if the dopants were dispersed homogeneously within the polymer matrix.

Optical Absorption and ESR Spectra of $\mathrm{SO}_{3}$ Doped PPS under Photoirradiation

To make clear whether the electronic structures derived from the optical absorption and ESR spectra are the same or not, the change of these spectra under photoirradiation was investigated.

For measurements of optical absorption and ESR spectra of $\mathrm{SO}_{3}$-doped PPS under photoirradiation, UV-light and near IR-light corresponding to the absorption bands at 3.1 and $1.0 \mathrm{eV}$ (Figure 1), respectively, were used as the photoexcitation source. Figure 4 shows the ESR spectra of $\mathrm{SO}_{3}$-doped PPS $\left(8.9 \mathrm{~mol}^{\circ} \%\right.$ $\mathrm{SO}_{3}$ ) before (broken line) and after UV-light irradiation (solid line). The intensity of ESR signals was enhanced with irradiation time, while linewidth and lineshape were unchanged. ESR signals reached a steady state after irradiation for $c a .15$ minutes and these intensities 


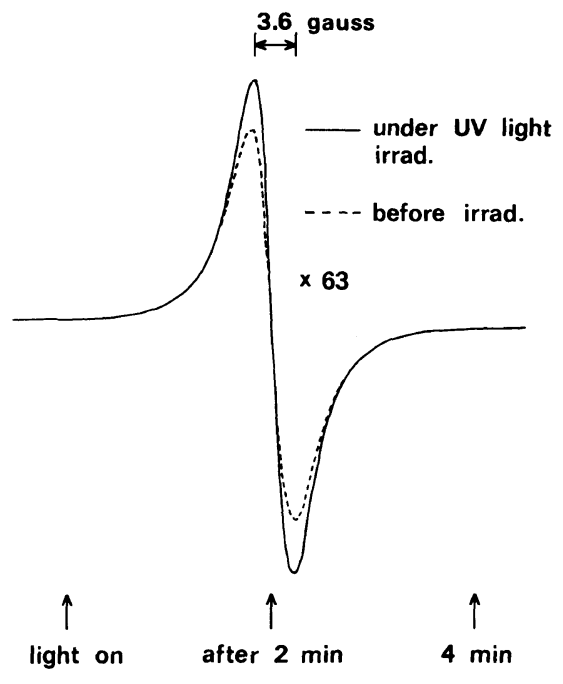

Figure 4. ESR spectra of $\mathrm{SO}_{3}$-doped PPS before and after UV-light irradiation.

increased by $15-20 \%$ compared with that before irradiation. ESR spectra of $\mathrm{SO}_{3}$-doped PPS were little affected by near IR-light irradiation. Figure 5 exhibits the dark decay of ESR signals of $\mathrm{SO}_{3}$-doped PPS after the stop of UV-light irradiation. The ESR spectrum irradiated by UV-light for 15 minutes returned gradually to the initial state. The half-life period of the dark decay process was approximately 40 minutes. This change of ESR spectra by UV-light irradiation was reversible. Figure 6 shows the optical absorption spectra of $\mathrm{SO}_{3}$-doped PPS $\left(9.2 \mathrm{~mol}_{\%}^{\circ} \mathrm{SO}_{3}\right)$ before and after UV-light irradiation. The intensity of two absorption bands at 1.0 and $3.1 \mathrm{eV}$ increased slightly after 5 minutes of UV-light irradiation. These spectra also returned reversibly to the original state after nearly the same time as that of ESR spectra. No difference in absorption spectra of $\mathrm{SO}_{3}$-doped PPS as well as ESR spectra was observed by near IR-light irradiation.

It is clear that absorption and ESR spectral changes of $\mathrm{SO}_{3}$-doped PPS are closely related each other. These spectra of $\mathrm{SO}_{3}$-doped PPS were sensitive to UV-light, while insensitive to near IR-light. From these results the spectral

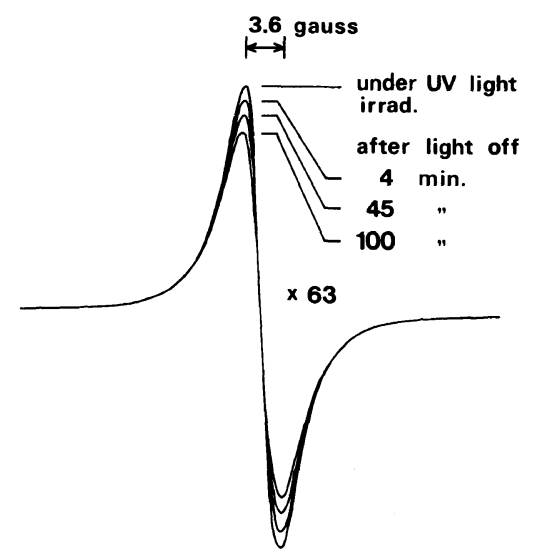

Figure 5. ESR spectral change of PPS- $\mathrm{SO}_{3}$ system in the dark after interruption of UV-light irradiation.

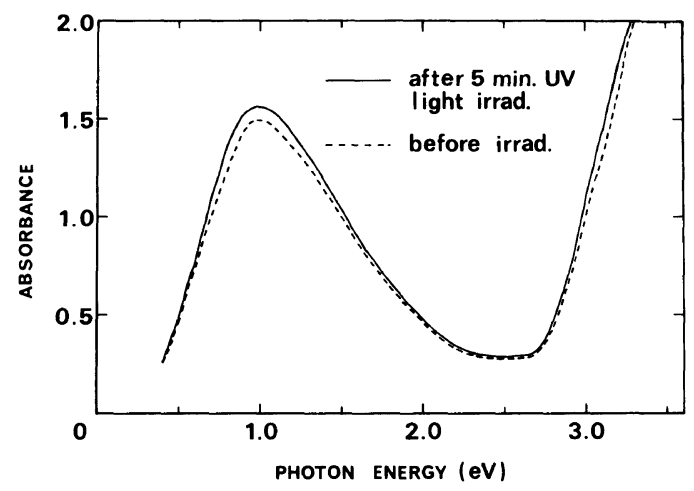

Figure 6. Absorption spectra of $\mathrm{SO}_{3}$-doped PPS before and after UV-light irradiation.

changes under photoirradiation are considered to be derived from the additional formation of radical-cations by the UV-light excitation. These additional radical-cations would be formed between PPS and $\mathrm{SO}_{3}$ although the detailed oxidation mechanism of $\mathrm{SO}_{3}$ molecules is not clear (for example $\mathrm{SO}_{3}{ }^{2-}, \mathrm{SO}_{4}{ }^{2-}$, and $\mathrm{S}_{2} \mathrm{O}_{5}{ }^{2-}$, etc.).

There are two possible regions in which additional radical-cations are formed by photo-doping process, where photo-doping means the increase in the spectral intensity by photoirradiation. One is unaltered parts of the PPS chain with doped $\mathrm{SO}_{3}$ molecules in that neighbourhood. The other is undoped parts in PPS where $\mathrm{SO}_{3}$ molecules are apart from the 


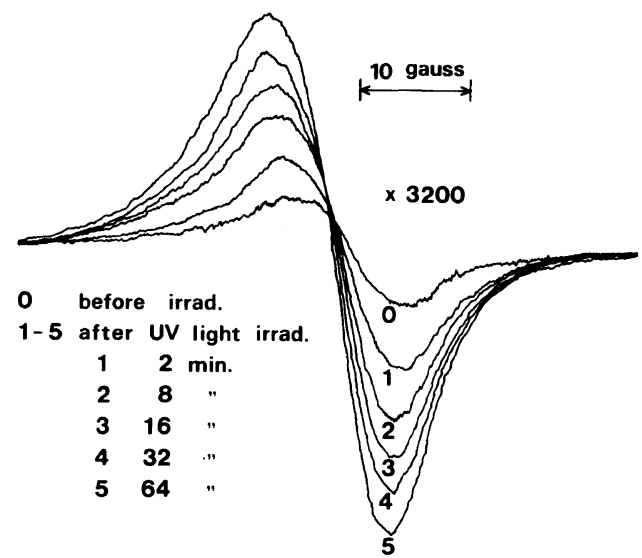

Figure 7. ESR spectral change of undoped PPS before and after UV-light irradiation.

\section{PPS chain.}

Optical Absorption and ESR Spectra of Undoped PPS under Photoirradiation

In order to elucidate the contribution of undoped parts of PPS to the photo-doping process, absorption and ESR spectra of undoped PPS were measured. Figure 7 exhibits the ESR spectra of undoped PPS before and after UV-light irradiation. The numeral $(\times 3200)$ in this figure indicates the amplification. In this case, modulation width multiplied by a factor of 10 compared with the case of $\mathrm{SO}_{3}$-doped PPS was used, since the ESR signal intensities of undoped ones were very weak. Therefore, we can make no reference to the lineshape and linewidth of ESR spectra of undoped PPS except the $g$-value. As shown in Figure 7, a very weak ESR signal with a $g$-value of 2.0046 was detected (curve No. 0). The origin of the radical species is yet unknown. On irradiating UV-light, the intensity of ESR signals of undoped PPS was enhanced with irradiating time. Those lineshapes changed to asymmetric as shown in this figure. After $c a$. 1 hour photoirradiation, the ESR signal reached the steady state and the $g$-value of that signal was 2.0060 . The spin density in the spectrum of photoirradiation (curve No. 5) was roughly estimated to

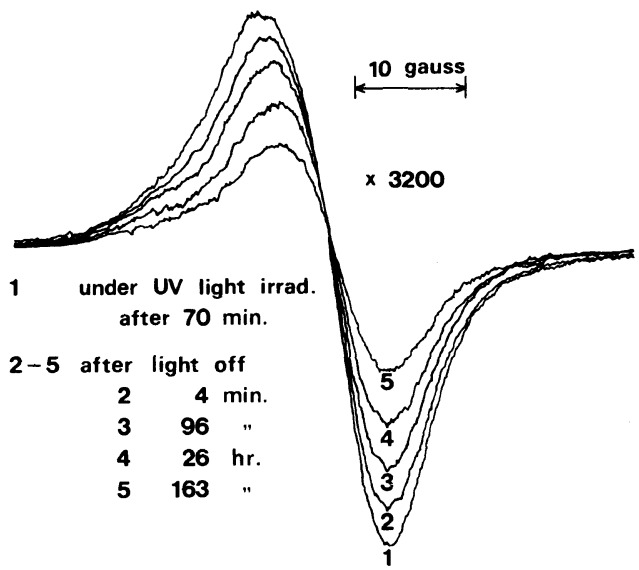

Figure 8. ESR spectral change of undoped PPS in the dark after the interruption of UV-light irradiation.

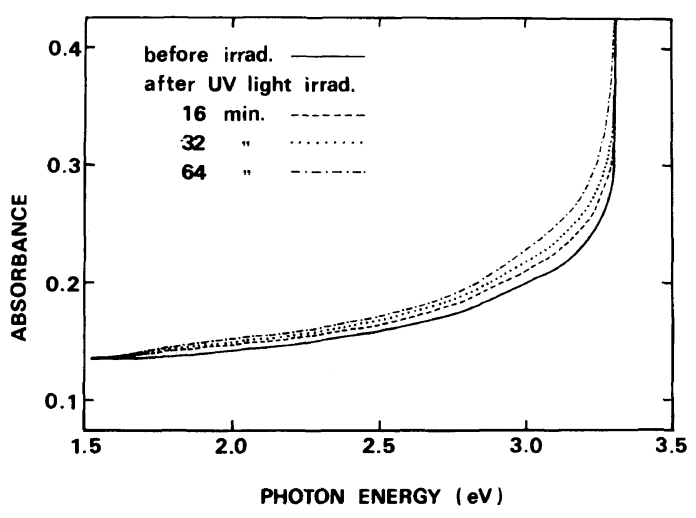

Figure 9. Absorption spectral change of undoped PPS before and after UV-light irradiation.

be $c a .1 .3 \times 10^{-4}$ spins/monomer unit. The ESR spectrum of undoped PPS was unchanged by the near IR-light irradiation as well as that of $\mathrm{SO}_{3}$-doped PPS.

The dark decay of the ESR spectrum of undoped PPS after the interruption of photoirradiation $(70 \mathrm{~min})$ is shown in Figure 8 . The ESR signals were very slowly reduced in the dark with a half-life period of $c a .80$ hours and did not completely return to the initial state. It was noted that the $g$-value of each spectra observed through the dark decay process was 2.0060. Figure 9 shows the absorption spectra of undoped PPS before and after UV-light irradiation. As shown in this figure, a small 
increase in absorbance of the region of about 1.5 to $3.3 \mathrm{eV}$ was observed after a relatively long time of photoirradiation. These spectra changed in the dark in the same manner as that of ESR spectra. The irradiation of near IRlight hardly contributed to the absorption spectral change of undoped PPS as well as the ESR spectrum.

These results suggest that UV-light irradiation has an effect on the formation of radical species. These long-life radical species indicate the presence of a weak charge-transfer structure. Therefore the origin of ESR signals with the $g$-value of 2.0060 seemed to be the weak charge-transfer structure formed between the sulfur atoms of PPS and oxygen atoms, where it is well-known that oxygen atoms enchance the dark DC conductivity of PPS film. Moreover, the irreversible spectral change of undoped PPS occurred by prolonged UV-light irradiation. In this case, the interchain crosslinking bridged by oxygen molecules seems to be responsible for the irreversible reaction. As described above, both undoped PPS and $\mathrm{SO}_{3}$-doped PPS were sensitive to UV-light excitation, but the electronic structure of undoped PPS which was formed by photoirradiation obviously differed from that of $\mathrm{SO}_{3}$-doped PPS. These results suggest that the formation of additional radical-cations on doped PPS chain by photoirradiation occurs in the unaltered parts of PPS in the vicinity of $\mathrm{SO}_{3}$ molecules.

\section{Electronic Structure of $\mathrm{SO}_{3}$-Doped PPS}

In this section, the electronic structure of $\mathrm{SO}_{3}$-doped PPS is discussed based on the results of optical absorption spectra, ESR spectra, and their changes with photoirradiation.

Although the optical absorption spectra are not inconsistent with the bipolaron mod$\mathrm{el}^{12-16}$, ESR spectra with the $g$-value of 2.0075 suggested the formation of sulfurcentered radical-cations. In addition, the spin density of $\mathrm{SO}_{3}$-doped PPS was enhanced mo- notonously with increase in the dopant concentration. These results of magnetic properties are in conflict with the presence of a bipolaron state. The formation of bipolarons in PPS- $\mathrm{SO}_{3}$ system would require the delocalization of sulfur-centered radicals over the adjacent phenyl ring resulting in the formation of a quinoid structure. However, neither the long conjugation over 5-6 phenyl rings nor the formation of continuous quinoid structure such as that of acceptor-doped PPP is reasonable for the PPS-acceptor system. Although either the isolated quinoid structure or the alternate quinoid one is considered as the possible bipolaron state for PPS-acceptor system, ${ }^{4}$ these electronic structures would occur at very high energy levels and not be formed within the band gap. As mentioned previously, the ESR spectrum with a $g$-value of 2.00737 was observed for di- $p$-tolyl sulfide as a monomer unit of PPS and assigned to radical-cation by Shine $e t$ al. ${ }^{8}$ They have also reported the absorption spectrum of the diphenyl sulfide in which two absorption bands have been observed at $1.50 \mathrm{eV}(825 \mathrm{~nm})$ and $2.68 \mathrm{eV}(460 \mathrm{~nm})$. These results suggest that nearly the same electronic structure as that of $\mathrm{SO}_{3}$-doped PPS was formed in the diphenyl sulfide in which the quinoid structure was prevented from forming. The quinoid structure as the bipolaron model is not necessarily formed in the PPS- $\mathrm{SO}_{3}$ system. A possible structure for the formation of sulfur-centered radical-cation on PPS chain doped with electron acceptor (A) is shown in Figure 10.

Brédas et al. calculated the electronic structure of PPS by using the valence effective Hamiltonian technique and showed the absolute positions and the band width of the three physically most interesting upper bands. ${ }^{17}$ However, they did not mention the excited states and the excitation energies. According to their results, the highest band of these three occupied bands (HOMO in Figure $11(\mathrm{~A}))$ is $1.17 \mathrm{eV}$ wide and has mainly contribu- 


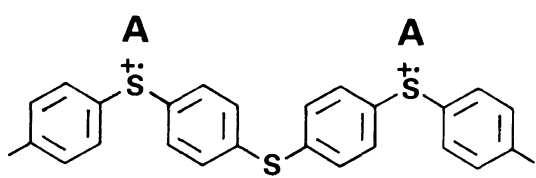

Figure 10. A possible structure for the formation of sulfur-centered radical-cations on the PPS chain doped with an electron acceptor (A).

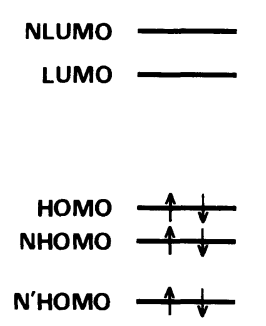

(A)

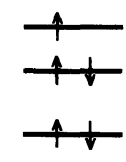

(B)

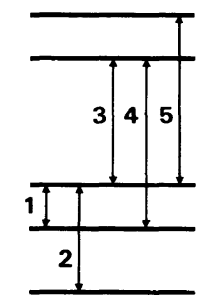

(C)
Figure 11. Energy level diagram of the electronic structure of undoped PPS (A) and radical-cation (B), and possible electronic transitions (C) originating from the state (B): HOMO and LUMO denote the highest occupied molecular orbital and the lowest unoccupied molecular orbital, respectively.

tions from $3 \mathrm{p}_{\mathrm{z}}$ sulfur and $2 \mathrm{p}_{\mathrm{z}}$ carbon orbitals (where $z$ is the direction perpendicular to the plane containing the sulfur atoms). The flatness $(0.14 \mathrm{eV}$ width) of the next higher band (NHOMO in Figure 11(A)) which gives rise to a very high peak in the density of states is due to the very small contributions coming from the sulfur atoms, as well as the carbon atoms bonded to sulfur. The lowest band ( $\mathrm{N}^{\prime}$ HOMO) in Figure 11(A) is $1.6 \mathrm{eV}$ wide and is very similar to the band HOMO, but with bonding interactions between sulfur atoms and their neighbors. The absolute positions of these bands are $-8.62 \mathrm{eV}$ at $k=\pi / a$ for HOMO, $-9.33 \mathrm{eV}$ for NHOMO, and -10.69 $\mathrm{eV}$ for $\mathrm{N}^{\prime} \mathrm{HOMO}$, respectively. Therefore, energy differences between the HOMO and the lower bands are 0.71 and $2.07 \mathrm{eV}$, respectively. The removal of one electron by acceptor doping seems to give rise to the singly occupied HOMO (see Figure 11(B)), which results in a sulfur-centered radical-cation.
The energy level of the singly occupied HOMO would be raised compared with that of fully occupied HOMO, since the singly occupied band is relatively unstable and the lability of the radical-cation causes the conformational change. The singly occupied HOMO would permit the electronic transitions shown in Figure 11(C). In this figure, only five allowed transitions are taken into account in the range of UV to near IR regions. The absorption bands of $\mathrm{SO}_{3}$-doped PPS observed at 0.9 and $3.1 \mathrm{eV}$ (see Figure 1) are considered to be assigned to the electronic transitions of No. 1 and No. 2 in Figure 11(C), respectively. The above-mentioned electronic configuration of radical-cation is in reasonable accord with not only the results of magnetic properties such as the $g$-values of ESR signals and the spin density change during doping but also those of optical properties.

Absorption and ESR spectral changes of $\mathrm{SO}_{3}$-doped PPS under photoirradiation are due to the additional formation of radicalcations by UV-light excitation. Since the transfer of electrons which are located in the singly occupied HOMO through the photoexciting leads to decrease the spin density, this mechanism does not occur. The electron transfer from unaltered parts of PPS to dopant molecules seems to contribute to the mechanism of photo-doping.

\section{CONCLUSION}

The intensity of two absorption bands of PPS- $-\mathrm{SO}_{3}$ system observed at 0.9 and $3.1 \mathrm{eV}$ increases with the rise of doping level. The intensity of ESR signals of $\mathrm{SO}_{3}$-doped PPS with the $g$-value of 2.0075 goes up with increase in the doping level as well as optical absorption, while the linewidth shows a nearly constant value of 3.6 Gauss. The spin density or the number of spins per monomer unit monotonously increases with the rise of $\mathrm{SO}_{3}$ concentration, and reaches 0.623 at 105.9 $\mathrm{mol} \%$ doping. 
Absorption and ESR spectra of $\mathrm{SO}_{3}$-doped PPS are closely associated with each other through the processes of chemical doping and photoirradiating. Therefore, both spectra are considered to originate from the same electronic structure. From a comparison of the experimental results with the theoretical models, spectroscopic observations suggest the presence of radical-cations on the sulfur atoms of PPS rather than the bipolaron state. Two intense bands of the PPS- $\mathrm{SO}_{3}$ system observed at 0.9 and $3.1 \mathrm{eV}$ are considered to be assigned to electronic transitions from low lying occupied bands to the singly occupied highest band.

Absorption and ESR spectral changes of $\mathrm{SO}_{3}$-doped PPS under photoirradiation are considered to be due to the additional formation of radical-cations by UV-light excitation. The electron transfer from unchanged parts of PPS to dopant molecules seems to contribute to the mechanism of photo-doping.

The UV-light irradiation of undoped PPS results in long-life radical species responsible for the ESR signal with a $g$-value of 2.0060 . The long-life species would indicate the presence of a weak charge-transfer structure formed between sulfur atoms of PPS and oxygen atoms.

Acknowledgement. Thanks are due to Kureha Chemical Industry Co., Ltd. for kindly supplying the transparent PPS films.

\section{REFERENCES}

1. J. E. Frommer, R. L. Elsenbaumer, and R. R. Chance, "Polymers in Electronics," T. Davidson, Ed., American Chemical Society, Symposium Series No. 242, Washington, 1984, p 447.

2. T. C. Clarke, K. K. Kanazawa, V. Y. Lee, J. F. Rabolt, J. R. Reynolds, and G. B. Street, J. Polym. Sci., Polym. Phys. Ed., 20, 117 (1982).

3. L. W. Shacklette, R. L. Elsenbaumer, R. R. Chance, H. Eckhardt, J. E. Frommer, and R. H. Baughman, J. Chem. Phys., 75, 1919 (1981).

4. R. H. Friend and J. R. M. Giles, J. Chem. Soc., Chem. Commun., 1101 (1984).

5. Y. Tanabe, H. Shimizu, and H. Kanetsuna, Rep. Prog. Polym. Phys. Jpn., 28, 381 (1985).

6. H. Shimizu, Y. Tanabe, and H. Kanetsuna, Polym. J., 18, 367 (1986).

7. R. R. Chance, R. H. Baughman, J. L. Brédas, H. Eckhardt, R. L. Elsenbaumer, J. E. Frommer, L. W. Shacklette, and R. Silbey, Mol. Cryst. Liq. Cryst., 83, 1249 (1982).

8. H. J. Shine, M. Rahman, H. Seeger, and G. S. Wu, J. Org. Chem., 32, 1901 (1967).

9. L. D. Kispert, L. A. Files, J. E. Frommer, L. W. Shacklette, and R. R. Chance, J. Chem. Phys., 78, 4858 (1983).

10. D. P. Murray, L. D. Kispert, and J. E. Frommer, $J$. Chem. Phys., 83, 3681 (1985).

11. M. Kuroiwa, K. Maeda, S. Tasaka, S. Miyata, T. Hashimoto, and A. Takaku, Kobunshi Ronbunshu, 41, 63 (1984).

12. J. L. Brédas, R. R. Chance, and R. Silbey, Phys. Rev., B, 26, 5843 (1982).

13. K. Fesser, A. R. Bishop, and D. K. Campbell, Phys. Rev., B, 27, 4804 (1983).

14. J. H. Kaufman, N. Colaneri, J. C. Scott, and G. B. Street, Phys. Rev. Lett., 53, 1005 (1984).

15. J. L. Brédas, J. C. Scott, K. Yakushi, and G. B. Street, Phys. Rev., B, 30, 1023 (1984).

16. J. C. Scott, J. L. Brédas, K. Yakushi, P. Pfluger, and G. B. Street, Synthetic Metals, 9, 165 (1984).

17. J. L. Brédas, R. R. Chance, R. Silbey, G. Nicolas, and Ph. Durand, J. Chem. Phys., 77, 371 (1982). 